\title{
Monatsschrift für
}

\section{Kriminologie}

und Strafrechtsreform

Sonderdruck 
Die internetbasierte Opferbefragung als Instrument der Dunkelfeldforschung - Grenzen und Chancen

von Angelika Treibel und Joachim Funke

Zusammenfassung

Das Internet wurde als Instrument viktimologischer Dunkelfeldforschung bisher wenig erforscht. Im vorliegenden Artikel geht es darum, die Chancen und Grenzen des Internets in Bezug auf die Durchührung von Opferbefragungen - insbesondere von Opfern sexueller Gewalt - zu beleuchten und kritisch zu bewerten. Die vielfältigen Vorteile und Möglichkeiten internetbasierter Befragungen werden vor allem durch methodische Probleme eingeschränkt, wie beispielsweise die Unkontrollierbarkeit der Erhebungsbedingungen und die Nicht-Repräsentativität der Internet-Nutzenden für die Ge amtbevölkerung. Die Ergebnisse einer Online-Befragung von Opfern sexueller Gewalt verdeut(lie ichen Möglichkeiten und Grenzen des Mediums. Die Notwendigkeit weiterer Forschung auf diesem Gebiet wird aufgezeigt.

Die repräsentative Opferbefragung als Instrument der Dunkelfeldforschung

Ziel der Dunkelfeldforschung ist es, möglichst umfassende und zuverlässige Daten darüber zu erlangen, wie hoch die Anzahl der Delikte ist, die den Strafverfolgungsbehörden nicht angezeigt wurden. Repräsentative Opferbefragungen, die in der Bundesrepublik Deutschland in unregelmäßigen Abständen durchgeführt werden, sind eines der wichtigsten Instrumente dieser Dunkelfeldforschung.

Das Dunkelfeld bei den Straftaten gegen die sexuelle Selbstbestimmung ist im Vergleich zu anderen Delikten besonders groß (vgl. Schneider 1998, 322 f.). Die Dunkelziffe steigt noch einmal, wurde das Delikt im sozialen Nahraum begangen, was bei der überwiegenden Mehrzahl dieser Delikte der Fall ist. Viele der Opfer teilen sich niemandem mit, und es ist davon auszugehen, dass sie es auch im Rahmen einer Opferbefragung nicht tun (vgl. Wetzels \& Pfeiffer 1995, 2). Hierdurch ist die Aussagekraft von repräsentativen Opferbefragungen eingeschränkt. Weiter einschränkend ist, dass bestimmte Personengruppen in »repräsentativen « Stichproben unterrepräsentiert sind, gleichzeitig aber ein erhöhtes Risiko haben, Opfer von Gewalttaten, auch von sexueller Gewalt, zu werden, z.B. Prostituierte, in Heimen lebende Personen sowie Inhaftierte. So können insgesamt auch die auf Grundlage der repräsentativen Opferbefragungen angestellten Schätzungen nur ungefähre Werte liefern (zur grundsätzlichen Problematik von Opferbefragungen siehe Wetzels 1996); gleichwohl stellen sie die beste zur Verfügung stehende Methode dar, um die Größe des Dunkelfeldes bei sexueller Gewalt zu schätzen. Repräsentative Opferbefragungen werden in der Bundesrepublik Deutschland in Form von Face-to-Face-Interviews, schriftlichen Befragungen, Telefonbefragungen oder einer Kombination dieser $\mathrm{Me}-$ thoden durchgeführt (vgl. Baurmann, Hermann, Störzer E Streng 1991).

Der vorliegende Artikel befasst sich mit einem weiteren möglichen Medium zur Durchführung von Opferbefragungen: dem Internet. Hierbei geht es um zwei Fragestellungen: Inwieweit ist eine internetbasierte Opferbefragung durchführbar und sinnvoll

1. im Sinne der quantitativen Dunkelfeldforschung und

2. im Sinne einer qualitativen Opferbefragung (z.B. über die Nutzung von Hilfeeinrichtungen, die Lebenssituation der Opfer, ihr Copingverhalten etc.)?
Betrachten wir zunächst Punkt 1, das primäre Ziel repräsentativer Opferbefragungen, nämlich die Erlangung valider Daten, auf deren Grundlage die Größe des Dunkelfeldes geschätzt werden kann. Repräsentative Opferbefragungen, die in Form von Face-to-FaceInterviews an großen Stichproben durchgeführt werden, bringen einen erheblichen zeitlichen und personellen Aufwand mit sich; dies gilt in geringerem Ausmaß ebenfalls für repräsentative Telefonbefragungen (vgl. Baurmann u.a. 1991).

Potenziale der Online-Befragung

Wie verhält es sich mit Online-Befragungen? Eine Online-Befragung in Form eines WWW-Fragebogens bietet auf den ersten Blick ideale Voraussetzungen, um einige Probleme zu lösen, die Face-to-Face- oder Telefon-Interviews in einer großen Stichprobe zu einem sensiblen Thema mit sich bringen (vgl. hierzu auch Batinic 2001):

- Die mögliche Stichprobengröße einer Online-Befragung geht über die Möglichkeiten von Face-to-Face- oder Telefon-Interviews weit hinaus - zumindest theoretisch Nach der aktuellen ARD/ZDF-Online-Studie für das Jahr 2003 sind 53,3\% der Bundesdeutschen online, dies entspricht 34,3 Millionen Personen ab 14 Jahren (van Eimeren, Gerhard E Frees 2003).

- Eine Befragung über das Internet in Form eines Online-Fragebogens bietet eine Anonymität, die bei Face-to-Face-Befragungen nicht gegeben ist und über die Anonymität der Telefonbefragung weit hinausgeht.

- Die Bekanntmachung der Befragung und die Erhebung der Daten sind unabhängig von Zeit und Ort.

- Personen, die an der Befragung interessiert sind, haben die Möglichkeit, zu einem selbst bestimmten Zeitpunkt an dieser teilzunehmen, und sie können die Entscheidung zur Teilnahme jederzeit rückgängig machen.

- Eine Online-Befragung bietet eine hohe Objektivität, weil Interviewer-Effekte ausgeschlossen werden können.

- Eine Online-Befragung ist ökonomisch: Im Vergleich zu Face-to-Face-Interviews oder Telefonbefragungen können erhebliche personelle, materielle und finanzielle Ressourcen eingespart werden.

- Die Daten liegen bereits in elektronischer Form vor, was die Auswertung erleichtert.

- Das Internet bietet die Möglichkeit, die Methode der randomisierten Antworttechnik (randomized response technique, RRT), für deren Anwendung große Stichproben notwendig sind, effizient einzusetzen.

Die RRT ist ein quantitatives Verfahren, das sich bei der Erhebung von sensiblen und tabubesetzten Themen als zuverlässige Methode erwiesen hat, um die Wahrscheinlichkeit wahrheitsgemäßer Angaben zu erhöhen, sowohl bei Opfern als auch bei Tätern. Die Methode, die in unterschiedlichen Variationen durchführbar ist, basiert im Prinzip darauf, dass als Resultat eines Randomisierungsprozesses nur ein Teil der an der Befragung teilnehmenden Personen aufgefordert wird, die »sensible« Frage zu beantworten, die anderen beantworten eine themenunspezifische Frage. Welche Teilnehmer oder Teilnehmerinnen die "sensible« Frage beantwortet haben, ist unbekannt, so dass ein Maximum an Anonymität gewährleistet ist. Die Berechnungen basieren dann auf den - bekannten - Wahrscheinlichkeiten, mit denen die "sensible« bzw. die themenunspezifische Frage beantwortet wurden. Musch, Bröder $\&$ Klauer (2001) wandten die RRT erstmals online an und konnten zeigen, dass diese Methode auch in der anonymen Erhebungssituation des Internets noch einen nachweislichen Effekt zeigte: Die wahrgenommene (und tatsächliche) Anonymität der Befragung wurde 
durch Anwendung der RRT erhöht, was dazu führte, dass die Bereitschaft der Teilnehmenden stieg, illegales Verhalten zuzugeben.

Grenzen der Online-Befragung

Den genannten Potenzialen internetbasierter Online-Befragungen stehen grundlegende methodische Einschränkungen und Grenzen entgegen, hat man eine repräsentative $\mathrm{OP}$ ferbefragung zum Ziel:

- Es gibt keine systematische Dokumentation der Internet-Nutzenden, vergleichbar mit Telefon- oder städtischen Adressverzeichnissen. Das Ziehen einer repräsentativen Stichprobe ist - über das Internet - unmöglich. Die theoretische Möglichkeit, zufällig generierte E-Mail-Adressen als Grundlage zu nehmen, ist neben der Fragwürdigkeit der Methode auch insofern problematisch, als eine Anfrage per E-Mail eine von den Empfängern und Empfängerinnen u.U. nicht gewünschte Nachricht darstellt und somit unter die Problematik des Spamming fällt.

Die Verbreitung des Internets ist zwar groß, es fallen jedoch bestimmte Personengruppen systematisch aus: ältere, formal weniger hoch gebildete und nicht berufstätige Personen sind unterrepräsentiert; auch das Geschlechterverhältnis von $56 \%$ männlichen und $44 \%$ weiblichen Internet-Nutzenden ist nicht repräsentativ für die bundesdeutsche Bevölkerung (van Eimeren u.a. 2003).

Schließlich ist die Anonymität des Internets die große Chance, gleichzeitig aber auch eines der größten Probleme einer internetbasierten Opferstudie, befinden sich sowohl die Erhebungsbedingungen als auch die Teilnehmenden vollständig außerhalb jeder Erfassbarkeit oder Kontrollierbarkeit der Untersuchungsdurchführenden. Es gibt - im Gegensatz zu »Real-Life «-Befragungen - die Gefahr der mehrfachen Teilnahme. Zwar ist eine Mehrfachteilnahme teilweise über die Kontrolle der IP-Adressen möglich, dies jedoch nur in begrenztem Umfang (Janetzko 1999, 203). Weiter ist zu bedenken, dass jede Form der Kontrolle die Anonymität relativiert, und sei es nur im subjektiven Empfinden der Teilnehmenden.

Es gibt keine zentrale Einrichtung im Internet, durch die Opfer und Nicht-Opfer mit relativ berechenbarer Wahrscheinlichkeit erreicht werden könnten (etwa vergleichbar mit der »Tagesschau « im Fernsehen). Das Nutzungsverhalten ist sehr unterschiedlich und die meisten Internet-Nutzenden bewegen sich kaum über ihre $₫$ Stammseiten « im Internet hinweg (van Eimeren u.a. 2003, 345).

Weiter ist es unwahrscheinlich, dass Personen, die nicht Opfer einer Straftat wurden, motiviert sind, an einer Befragung teilzunehmen, in der es um Opferwerdung geht; auch insofern wird eine Online-Befragung, deren Teilnehmer und Teilnehmerinnen ausschließlich über das Internet rekrutiert werden, stark selbst selektiert sein.

Was die bereits erwähnte RRT betrifft, so muss hier zunächst festgehalten werden, dass sie in Form einer Opferbefragung online noch nicht durchgefübrt wurde. Inwieweit die Ergebnisse der Online-Anwendung von Musch et al. (2001), in der »Täterverhalten « in einem ganz anderen Kontext, nämlich dem der Steuerhinterziehung erhoben wurde, auf die Online-Befragung von Opfern sexueller Gewalt übertragen werden kann, muss an dieser Stelle unbeantwortet bleiben. Eine der wenigen Studien, in der die RRT im Sinne der Opferforschung angewandt wurde, ist die (schriftliche) Befragung von Soeken \& Damrosch (1986): Sie hatten die RRT in einer Befragung angewandt, in der die Häufigkeit von Vergewaltigungen erhoben wurde. Die Viktimisierungsrate in der Untersuchung entsprach den geschätzten Größenverhältnissen des Dunkelfeldes bei Vergewaltigungen. Inwieweit die erfolgreiche Anwendung dieser Methode bei (potenziellen) Opfern auch in einer Online-Umgebung funktioniert, muss - insbesondere in Anbetracht der nicht unproblematischen Erhebungsbedingungen im Internet - Gegenstand zukünftiger Studien sein. So hat die Gewährleistung völliger Anonymität nicht notwendigerweise bei Tätern und Opfern die gleiche Wirkung, liegen dem Schweigen der Opfer doch völlig andere psychologische Ursachen zu Grunde als dem Schweigen von Tätern.

Zusammenfassend lässt sich sagen, dass die Durchführbarkeit einer repräsentativen $\mathrm{OP}$ ferbefragung über das Internet grundsätzlich in Frage gestellt werden muss.

Denkbar wäre es, eine repräsentative Stichprobe nach herkömmlichen Methoden zu ziehen, die Bekanntmachung telefonisch oder postalisch durchzuführen und auf diesem Wege um Teilnahme an einer Online-Befragung zu bitten. So könnten die Potenziale des Mediums zumindest teilweise genutzt werden. Inwieweit der $*$ Transfer « von der postalischen oder telefonischen Bitte um Teilnahme hin zum Aufrufen der genannten Internetseite tatsächlich funktionieren könnte, muss Gegenstand zukünftiger Forschung sein und kann an dieser Stelle nicht beantwortet werden. Dies gilt ebenso für die anzunehmende Verzerrung der Stichprobe, weitere systematische Ausfälle bestimmter Personengruppen sowie die Problematik der Teilnahmekontrolle etc.

Ergebnisse einer Online-Befragung von Opfern sexueller Gewalt

Ungeachtet dieser Einschränkungen und jenseits der Frage nach repräsentativen Stichproben verdienen internetbasierte Opferbefragungen Beachtung, womit wir bei Punkt 2, der Frage nach eher qualitativ angelegten Opferbefragungen via Internet, angekommen sind. Fest steht, dass das Internet von Opfern sexueller Gewalt in vielfältiger Form aktiv genutzt wird: zur Informationssuche, zum Austausch mit anderen Betroffenen, zur Inanspruchnahme professioneller Hilfe und zur Gestaltung eigener Homepages, in denen das eigene Opferwerden thematisiert wird. Die Erstautorin dieses Beitrags führte im Jahr 2002 eine Online-Befragung von Opfern sexueller Gewalt durch, in der es darum ging, etwas über das Nutzungsverhalten von Opfern sexueller Gewalt in Bezug auf themenbezogene Internetangebote zu erfahren. Weiter ging es darum, die Durchführbarkeit einer solchen Befragung im Internet grundsätzlich zu überprüfen.

Die Befragung, die in Form eines WWW-Fragebogens durchgeführt wurde, führte innerhalb der relativ kurzen Laufzeit von 27 Tagen zu 149 auswertbaren Fragebögen. Die Bewertung der Befragung durch die Teilnehmenden war insgesamt positiv, einige Teilnehmer und Teilnehmerinnen brachten explizit ihr Interesse an weiteren Befragungen zum Ausdruck. Eine Zusammenfassung der Befragungsergebnisse findet sich bei Treibel (2003). Die Ergebnisse sollen an dieser Stelle nur im Hinblick auf die für die Dunkelfeldforschung relevanten Aspekte betrachtet werden: Dunkelfeldforschung wurde im Rahmen der genannten Befragung insoweit durchgeführt, als nach Anzeige bzw. NichtAnzeige der Tat gefragt wurde. Die Ergebnisse stimmen mit den bisherigen Erkenntnissen der Dunkelfeldforschung überein: Es zeigte sich, dass die überwiegende Mehrzahl der Delikte $(83,2 \%, n=124)$ nicht angezeigt worden war und dass die Anzeigehäufigkeit mit dem Bekanntheitsgrad des Täters bzw. der Täterin abnahm.

Weiter wurden die Teilnehmenden gefragt, ob sie sich, und - gegebenenfalls - wem sie sich mitgeteilt haben. Es zeigte sich, dass es eine, wenn auch kleine Gruppe von Teilnehmern und Teilnehmerinnen gab $(4 \%, \mathrm{n}=6)$, die sich nach eigenen Angaben bisher ausschließlich über das Internet mit anderen ausgetauscht hatten. Alle diese Fälle lagen nicht überraschend - im Dunkelfeld. Das bedeutet, dass es Opfer gibt, für die die Kommunikation via Internet niedrigschwelliger ist als jede andere Form der Kontaktaufnahme. Es ist davon auszugehen, dass diese Opfer nur über das Internet »erreichbar« sind. 
Das Internet bietet im Sinne einer qualitativen Dunkelfeldforschung ohne Zweifel Potenziale: So können weitere Befragungen durchgeführt werden, die sich mit den Faktoren beschäftigen, die das Anzeigeverhalten der Opfer beeinflussen, die die Opfer zu ihren Erfahrungen mit bestimmten Institutionen befragen etc. Die bei der durchgeführten OnlineBefragung teilweise sehr ausführlichen Antworten der Teilnehmer und Teilnehmerinnen geben einen Hinweis darauf, dass zumindest ein Teil der Opfer bereit und in der Lage ist, im Rahmen einer Online-Befragung ausführlich über das eigene Erleben zu berichten Diese Form der qualitativen Dunkelfeldforschung mit offenen Fragestellungen ist im Vergleich zu quantitativ angelegten Fragebögen mit schnell anklickbaren Buttons weniger störanfällig im Sinne von Falschteilnahmen. Die Plausibilität der Antworten ist kontrollierbar, und es ist fraglich, inwieweit "Spaßteilnehmer« die Motivation aufbringen, Texte zu erfinden, die glaubhaft von Erfahrungen mit Hilfeeinrichtungen handeln. Sicher wäre die Motivation zur Falschteilnahme größer, ginge es um Fragen des Tathergangs. Eine solche Befragung ist nicht nur aus diesem Grunde problematisch: Fragen zum Tathergang sind eine hohe potenzielle Belastung für die Opfer und bedürfen entsprechender Vorsicht und der eventuellen Bereitstellung von Kriseninterventionsangeboten. Im Hinblick auf solche Belastungen hat sich im Internet unter den Betroffenen eine Selbsthilfekultur entwickelt, die darin besteht, auf möglicherweise belastende Inhalte durch Leerräume im Text frühzeitig hinzuweisen (Spoiler) bzw. eine Verfremdung der Wörter vorzunehmen, indem Buchstaben durch Sternchen (Splats) ersetzt werden (z.B. $\left.G^{*} w^{*} l t\right)$. Eine OnlineBefragung, die mögliche Belastungen der Opfer nicht thematisiert, könnte unter den Betroffenen entsprechend auf Ablehnung stoßen.

\section{Bilanz und Ausblick}

Zusammenfassend lässt sich sagen, dass die Chancen internetbasierter Opferbefragungen - derzeit - sicher eher im Bereich der qualitativen denn der quantitativen Forschung liegen. Repräsentative internetbasierte Opferbefragungen sind beim heutigen Stand von Technik und Organisation des Internets nicht erfolgversprechend durchführbar. Ungeachtet dessen bietet das Internet enorme Potenziale, um viktimologische Forschung durchzuführen. Die gezielte Befragung von Opfern zu ihren Erfahrungen mit Hilfeeinrichtungen, ihren Copingstrategien, ihren Lebensumständen und die Erforschung von Faktoren, die das Anzeigeverhalten beeinflussen, um nur einige Beispiele zu nennen, sind über das Internet sicher möglich. Die technischen Möglichkeiten des Internets bieten die Chance, weitere Methoden zu entwickeln und zu testen, wie beispielsweise die RRT Die Bedeutung der RRT für die viktimologische Online-Forschung kann derzeit noch nicht eingeschätzt werden. Es ist jedoch davon auszugehen, dass die RRT die Wahrscheinlichkeit wahrheitsgemäßer Angaben sowohl bei Tätern und Täterinnen als auch bei Opfern erhöht.

Die Chancen von Opferbefragungen im Internet müssen Gegenstand weiterer Forschung sein. Angesichts des großen Dunkelfeldes bei sexueller Gewalt und den hohen Belastungen, denen die Opfer ausgesetzt sind auf der einen Seite, und den enormen technischen und methodischen Möglichkeiten, die das Internet bietet, auf der anderen Seite stehen Sinn und Notwendigkeit dieser Forschung außer Frage.

\section{Summary}

Research is scarce regarding the Internet as an instrument to explore unreported crime from a victimological perspective. This article critically reflects the chances and limits of online victim surveys - especially concerning victims of sexual violence. The various advantages and potentials of internet based surveys primarily face methodological constraints, e.g. the uncontrollability of survey- participation and the internet-community not being representative for the total population. Results of an online survey of victims of sexual violence point up the medium's possibilities and limitations. Further research in this field is necessary.

\section{Literatur}

Batinic, B. (2001). Fragebogenuntersuchungen im Internet. Aachen: Shaker. - Baurmann, M.C., Hermann, D. Störzer, HU. \& Streng, F (1991). Telefonische Befragung von Kriminalitätsopfern: Ein neuer Weg ins Dunkelfeld? MschrKrim 74/3, 159-173. - Janetzko, D. (1999). Statistische Anwendunen im Internet. Daten in Netzumgebungen erheben, auswerten und präsentieren. München: Addison-Wesley-Longman. - Musch, J., Bröder, A. E Klauer, K.C. (2001). Improving Survey Research on the World-Wide Web Using the Randomized Response Technique, in: U.-D. Reips \& M. Bosnjak (eds.), Dimensions of Internet Science, 179-192. Lengerich: Pabst. - Schneider, H.J. (1998). Der gegenwärtige Stand der kriminologischen Opferforschung. MschrKrim 81/5, 316-344. - Soeken, K.L. E Damrosch S.P. (1986). Randomized Response Technique: Applications to Research on Rape. PsychoDreibel A. (2003). Hilfe im Internet? Ergebnisse einer ogy of Wor Online-Befagung ren, B., Gerbard, $H$. \& Frees, B. (2003). ARD/ZDF-Online-Studie 2003. Internetverbreitung in Deutschland: Unerwartet hoher Zuwachs. Media Perspektiven 8/2003, 338-358. - Wetzels, P. (1996). Kriminalität und Opfererleben: Immer öfter immer das Gleiche? Defizite und Perspektiven repräsentativer Opferbefragungen als Methode empirisch-viktimologischer Forschung in der Kriminologie. MschrKrim 79/1, 1-24. - Wetzels, P. \& Pfeiffer, C. (1995). Sexuelle Gewalt gegen Frauen im öffentlichen und privaten Raum - Ergebnisse der KFN-Opferbefragung 1992. Kriminologisches Forschungsinstitut Niedersachsen (KFN), Forschungsberichte Nr. 37. Hannover: KFN.

(Anschr. d. Verf.: Angelika Treibel und Prof. Dr. Joachim Funke, Psychologisches Institut der Universität Heidelber Hauptstr. 47-51,69117 Heidelberg; joachim.funke@psychologie.uniheidelberg.de) 